Article

\title{
Antisense Activity across the Nesp Promoter is Required for Nespas-Mediated Silencing in the Imprinted Gnas Cluster
}

\section{Charlotte J. Tibbit ${ }^{1,5}$, Christine M. Williamson ${ }^{1,6}$, Stuti Mehta ${ }^{1,7}$, Simon T. Ball ${ }^{1,8}$, Mita Chotalia $^{2,9}$, Wade T. Nottingham ${ }^{1,10}$, Sally A. Eaton ${ }^{1,11}$, Mohamed M. Quwailid ${ }^{1}$, Lydia} Teboul $^{3}$, Gavin Kelsey ${ }^{2,4}$ and Jo Peters ${ }^{1, *}$

1 MRC Harwell, Mammalian Genetics Unit, Harwell Campus, Oxfordshire OX110RD, UK;

E-Mails: m.quwailid@ har.mrc.ac.uk (M.M.Q.); charlotte.tibbit@dpag.ox.ac.uk (C.J.T.);

c.williamson@har.mrc.ac.uk (C.M.W.); stuti.mehta@wolfson.oxon.org (S.M.);

s.ball@har.mrc.ac.uk (S.T.B.); wadenottingham@google.com (W.T.N.);

S.Eaton@victorchang.edu.au (S.A.E.)

2 Epigenetics Programme, The Babraham Institute, Cambridge CB223AT, UK; E-Mails: gavin.kelsey@babraham.ac.uk (G.K.); mita.chotalia@googlemail.com (M.C.)

3 Mary Lyon Centre, MRC Harwell, Harwell Campus, Oxfordshire OX110RD, UK;

E-Mail: 1.teboul@har.mrc.ac.uk

4 Centre for Trophoblast Research, University of Cambridge, Cambridge CB23EG, UK

5 Current address: MRC Functional Genomics Unit, Department of Physiology Anatomy \& Genetics, Le Gros Clark Building, University of Oxford, South Parks Road, Oxford OX13QX, UK

6 Current Address: MRC Harwell, Harwell Campus, Oxfordshire OX110RD, UK

7 Current address: GI Division, Their 340, Massachusetts General Hospital, Harvard Medical School, Boston, MA 02114, USA

8 Current address: Mary Lyon Centre, MRC Harwell, Harwell Campus, Oxfordshire OX110RD, UK

9 Current address: Genome Function Group, MRC Clinical Sciences Centre, Imperial College London, Hammersmith Hospital Campus, London W120NN, UK

${ }^{10}$ Current address: West London Free School, 2 Bridge Avenue, Hammersmith, London W69JP, UK

11 Current address: Molecular, Structural and Computational Biology Division, Victor Chang Cardiac Research Institute, Darlinghurst, NSW 2010, Australia

* Author to whom correspondence should be addressed; E-Mail: j.peters@ har.mrc.ac.uk; Tel.: +44-123-583-5410.

Academic Editor: George A. Calin

Received: 8 October 2015/Accepted: 17 November 2015/ Published: 30 November 2015 


\begin{abstract}
Macro long non-coding RNAs (lncRNAs) play major roles in gene silencing in inprinted gene clusters. Within the imprinted Gnas cluster, the paternally expressed Nespas lncRNA downregulates its sense counterpart Nesp. To explore the mechanism of action of Nespas, we generated two new knock-in alleles to truncate Nespas upstream and downstream of the Nesp promoter. We show that Nespas is essential for methylation of the Nesp differentially methylated region (DMR), but higher levels of Nespas are required for methylation than are needed for downregulation of Nesp. Although Nespas is transcribed for over $27 \mathrm{~kb}$, only Nespas transcript/transcription across a $2.6 \mathrm{~kb}$ region that includes the Nesp promoter is necessary for methylation of the Nesp DMR. In both mutants, the levels of Nespas were extraordinarily high, due at least in part to increased stability, an effect not seen with other imprinted lncRNAs. However, even when levels were greatly raised, Nespas remained exclusively cis-acting. We propose Nespas regulates Nesp methylation and expression to ensure appropriate levels of expression of the protein coding transcripts Gnasxl and Gnas on the paternal chromosome. Thus, Nespas mediates paternal gene expression over the entire Gnas cluster via a single gene, Nesp.
\end{abstract}

Keywords: long non-coding RNA; antisense; genomic imprinting; epigenetic silencing; Nespas; Nesp; Gnas cluster

\title{
1. Introduction
}

Imprinted genes generally occur in clusters and most imprinted clusters contain at least one long non-coding RNA [1]. Parental specific expression of genes within a cluster is under the overall control of an imprinting control region (ICR). For four well characterised clusters, the ICR contains the promoter of an unusually long non-coding RNA (lncRNA) that is exclusively expressed from the paternal allele and runs antisense to at least one protein coding gene within the cluster. All four lncRNAs, Airn in the Igf2r cluster [2], Kcnqlotl in the Kcnq1 cluster [3], Nespas in the Gnas cluster [4] and Ube3aas in the Snrpn cluster [5,6] are required for silencing one or more protein coding genes within their respective clusters.

Gnas is a complex imprinted cluster with three protein coding genes, Nesp that encodes the neurosecretory protein NESP55, Gnas that encodes the stimulatory G-protein Gs $\alpha$ and Gnasxl that encodes extra-large forms of Gs $\alpha$ [7] (Figure 1). Appropriate levels of both Gnasxl and Gnas are required for normal growth and survival [8-10]. The ICR for the cluster is unmethylated and active on the paternally derived allele and contains the promoter for Nespas [11]. Nespas is paternally expressed and runs antisense to its maternally expressed protein coding sense counterpart Nesp. Both a macro form and several spliced forms up to $1.4 \mathrm{~kb}$ in size of Nespas RNA are known [12]. Nespas is transcribed through the promoter of Nesp, which lies within a somatic differentially methylated region (DMR), that acquires methylation on the paternal allele post fertilisation [13-15]. When Nespas is not expressed the Nesp, DMR remains unmethylated and Nesp is expressed on the paternal allele, therefore the primary role of Nespas may be to repress Nesp [11].Low levels of Nespas expression can downregulate Nesp on the paternal allele through chromatin modification in the absence of DNA methylation at the Nesp DMR 
[4]. It has been proposed that DNA methylation of the Nesp DMR is essential to stabilise silencing of Nesp long term [4].

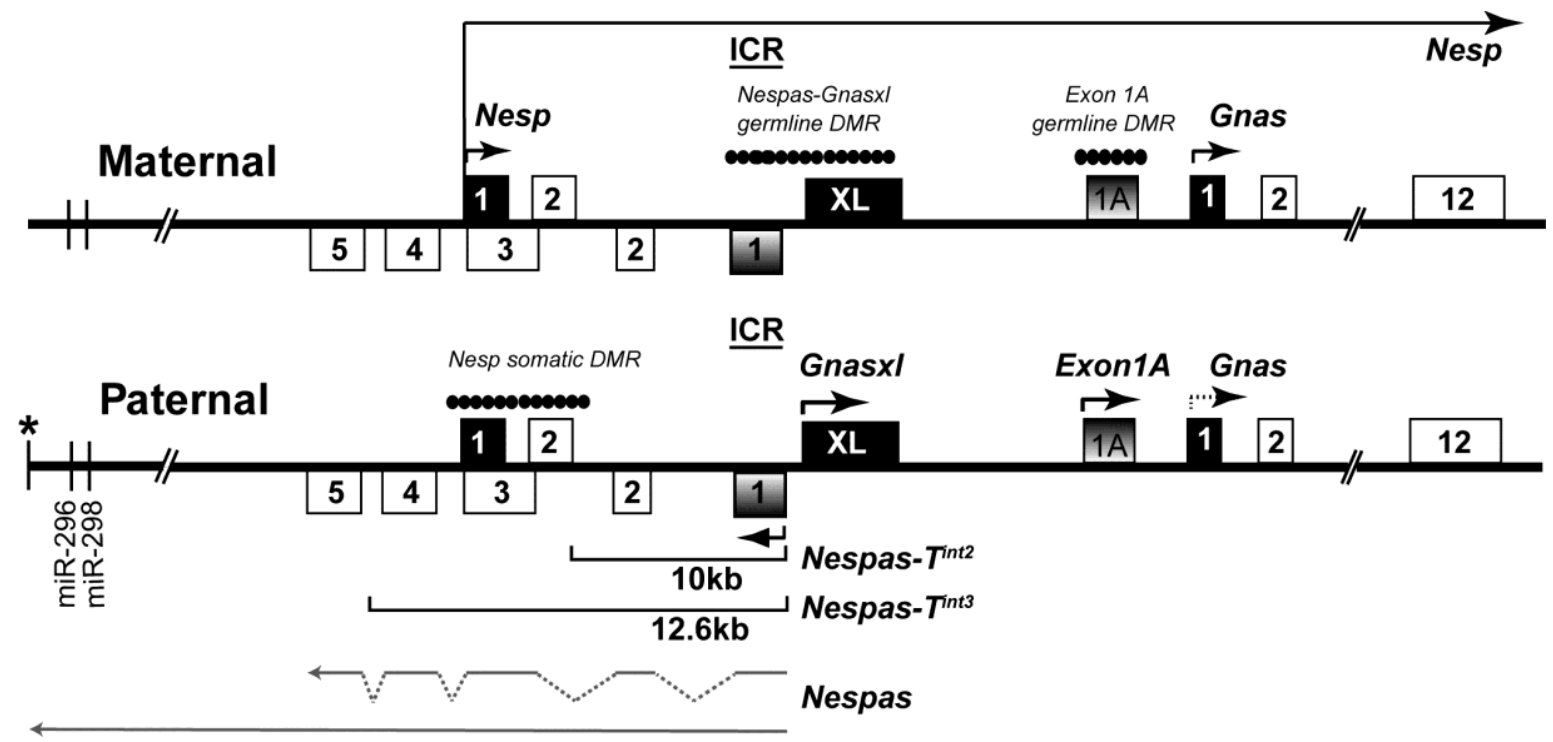

Figure 1. Organisation of the Gnas cluster. First exons of protein coding transcripts are shown as filled boxes and first exons of noncoding transcripts as shaded boxes. Arrows show the initiation and direction of transcription. Nesp is transcribed across the cluster. The arrow corresponding to the paternal Gnas allele is shown as a dotted line to indicate that the paternal Gnas allele is repressed tissue specifically. The $10 \mathrm{~kb}$ and $12.6 \mathrm{~kb}$ lines represent the new truncation mutations Nespas- $T^{\text {int2 }}$ and Nespas- $T^{\text {int3 }}$. Nespas non-coding transcripts are shown as grey lines. * represents the termination of Nespas transcription, approximately $30 \mathrm{~kb}$ from the Nespas promoter. Paternally expressed microRNAs are shown as vertical lines. Filled circles represent methylated regions. ICR represents the imprinting control region.

We have carried out the first study examining the effects of truncating an imprinted lncRNA to different lengths in an in vivo mouse model. Using two new truncation alleles, we show that expression of Nespas is required for methylation of the Nesp DMR. Our results show that a requirement for Nespas-mediated methylation and silencing in cis is that Nespas transcript/transcription needs to span the Nesp promoter. Although Nespas is transcribed for more than $27 \mathrm{~kb}$ [16,17], it is only Nespas transcript/transcription across a $2.6 \mathrm{~kb}$ region that includes the Nesp promoter that is required for methylation of the Nesp DMR. In both mutants, truncation of Nespas resulted in very highly elevated levels of truncated Nespas transcript, an effect not seen with truncation of other non-coding RNAs. However, even when Nespas levels were greatly raised Nespas remained exclusively cis-acting.

\section{Results}

\subsection{Nespas Transcription Extends over $30 \mathrm{~kb}$}

Previous work had shown evidence of Nespas transcription approximately $30 \mathrm{~kb}$ downstream from its promoter [16,17]. In the present study, using 3' RACE, we identified a poly(A) addition site, approximately $30 \mathrm{~kb}$ from the Nespas promoter and just downstream of a poly(A) signal AAUAAA, at 
nucleotide 122,587 (BAC sequence AL593857.10). This site represents the end of Nespas transcription as it aligns with the end of transcription in $12.5 \mathrm{dpc}$ placenta as determined by an RNA expression tiling array analysis using $50 \mathrm{bp}$ oligos spaced at 50 bp intervals [18].

\subsection{The Nesp DMR Is Unmethylated When Nespas Is Truncated before the Nesp Promoter}

To create the truncation mutant, Nespas- $T^{\text {int2 }}$ a polyadenylation cassette (pA) from the rabbit $\beta$-globin gene was inserted into Nespas intron 2 in an orientation (Ap) to truncate Nespas $10 \mathrm{~kb}$ from its promoter and downstream of the Nesp promoter (Figure 2a,b). Following paternal transmission, we determined whether Nespas was truncated by Taqman reverse transcription quantitative real-time PCR (RT-qPCR) analysis. An assay $3^{\prime}$ of the insertion with respect to Nespas transcription, specific to Nespas intron 4 and upstream of the Nesp promoter, detected an $80 \%$ reduction in Nespas level in $+/$ Nespas- $T^{\text {int } 2}$ (maternal allele precedes the paternal allele) relative to wild-type ( $n=6, P=1.0 \times 10^{-3}$; Figure $2 \mathrm{c}$ ). In contrast, an assay $5^{\prime}$ of the insertion with respect to Nespas transcription, specific for Nespas exon 1 spliced onto exon 2 and downstream of the Nesp promoter, detected an unexpectedly high 46 fold increase in Nespas in +/Nespas- $T^{i n t 2}$ compared with the level in wild-type $\left(n=6, P=1.5 \times 10^{-10}\right.$; Figure 2d). Thus, the low level of Nespas after the insertion compared with the high level before the insertion showed that most of the transcripts were truncated (as shown in the schematic in Figure 2e).

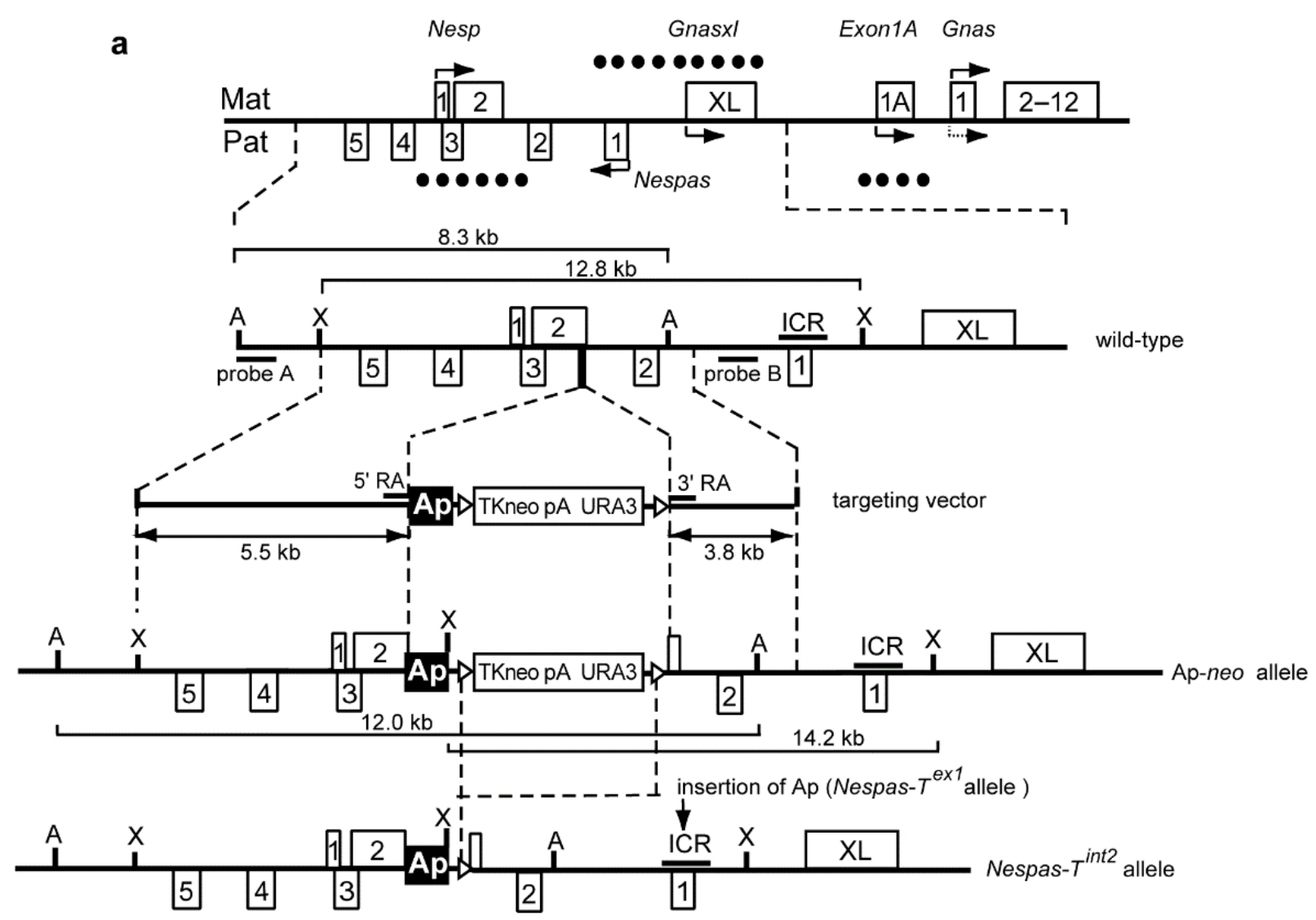

Figure 2. Cont. 


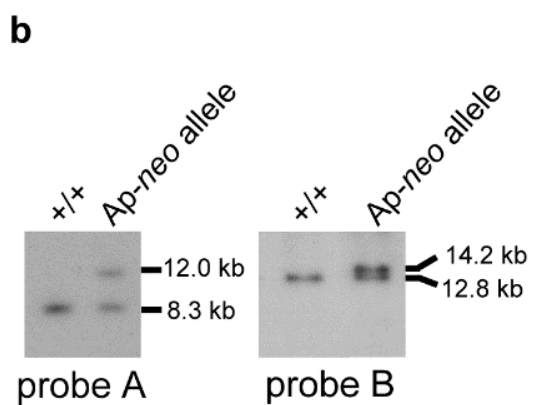

e

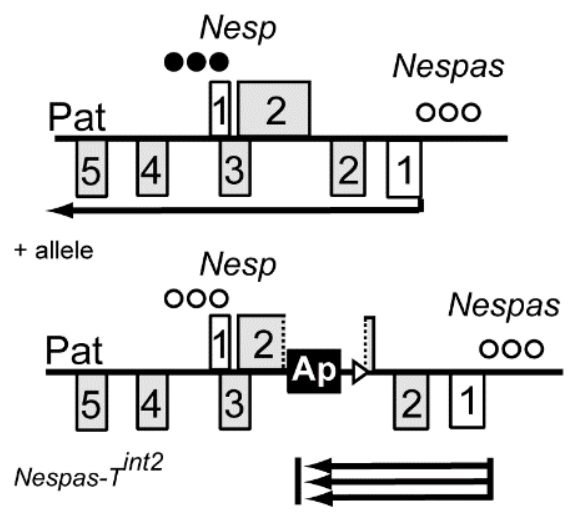

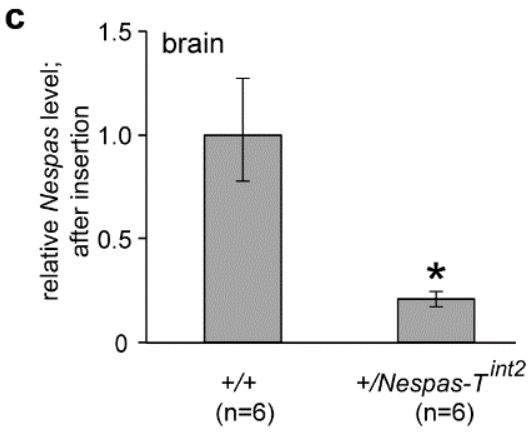

f Nesp DMR

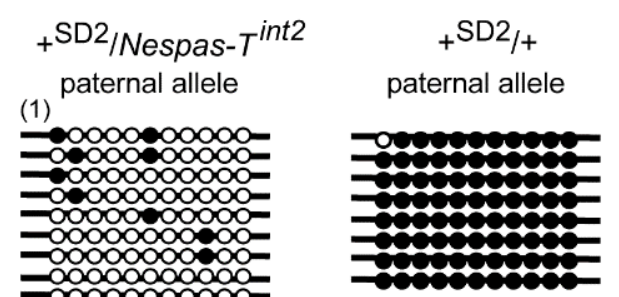



g

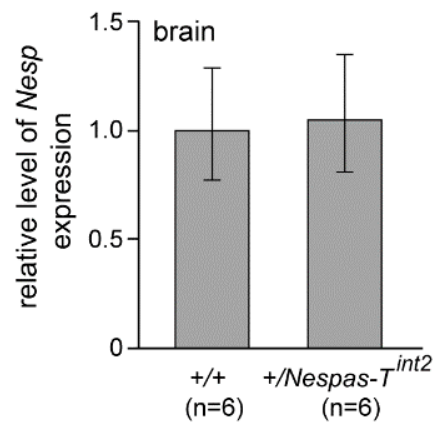

Figure 2. Truncation of Nespas before the Nesp promoter in Nespas intron 2/Nesp exon 2. (a) Schematic of the mouse Gnas locus showing the site of insertion of the rabbit $\beta$-globin polyadenylation cassette [2]. The targeting vector shows the location of a $1.2 \mathrm{~kb}$ fragment (black box labelled Ap) from the rabbit $\beta$-globin gene to truncate Nespas and the position of the selection cassette (TKneopA URA3), flanked by loxP sites (open triangles). The targeted allele was designated Nespas- $T^{\text {int } 2}$ allele after Cre-mediated excision of the selectable marker cassette. Methylated DMRs are shown by filled circles. A, AflII; X, XhoI; ICR, Imprinting Control Region. The arrow shows the approximate position of the polyadenylation cassette (pA) to truncate Nespas in exon 1 in Nespas-T $T^{e x 1}$ [4]; (b) Southern analysis of ES cell DNA showing correct targeting in the Ap-neo allele. Ap-neo targeted clones were identified by the presence of a $12.0 \mathrm{~kb}$ AflII fragment detected with the 5' external probe, probe A. Correct targeting at the 3 ' end was confirmed by the detection of a $14.2 \mathrm{~kb}$ XhoI fragment with Probe B; (c) Nespas is downregulated after the insertion; (d) Nespas is upregulated before the insertion; (e) Nespas is truncated and abundant in +/Nespas- $T^{\text {int2 }}$; (f) Bisulphite analysis showing the Nesp DMR is unmethylated on the paternal allele in ${ }^{\mathrm{SD} 2} / N e s p a s-T^{\text {int2 }}$. All clones from one individual are grouped into a block and two $+{ }^{\mathrm{SD} 2} / \mathrm{Nespas}-T^{\text {int2 }}$ individuals, (1) and (2), and one $+{ }^{\mathrm{SD} 2} /+$ individual were analysed. Each row of circles represents a clone derived from the paternal allele and each circle corresponds to a separate $\mathrm{CpG}$ (filled circle, methylated CpG; unfilled circle, unmethylated CpG) for nucleotides 140,450-140,800; (g) The Nesp level is unaffected in +/Nespas- $T^{\text {int2 }}$. 
We next investigated the methylation status of the paternal somatic DMR at Nesp. Bisulphite analysis was done on brain DNA from newborn offspring arising from crosses of Nespas-T $T^{\text {int } 2}$ carrier males with SD2 females carrying the Gnas imprinted region from Mus spretus. The presence of single nucleotide variants in the parents enabled the maternal and paternal alleles of Nesp to be distinguished. Figure $2 \mathrm{f}$ shows that the paternal Nesp DMR was unmethylated in $+/$ Nespas- $T^{\text {int } 2}$. A similar result was observed in +/Nespas- $T^{\text {int } 2}$ mice derived from a second independently targeted clone. Thus, the normally methylated paternal Nesp allele was unmethylated when Nespas was truncated before the Nesp DMR in $+/$ Nespas- $T^{\text {int2 }}$ (Figure 2e).

To investigate expression of Nesp from the mutant unmethylated paternal Nespas- $T^{\text {int } 2}$ allele, we measured transcript levels in newborn brain using a RT-qPCR Taqman assay specific for exon 1 spliced onto exon 2 of Nesp [4]. There was no significant difference in the level of Nesp transcript in newborn brain between wild-type and +/Nespas- $T^{\text {int2 }}$ (Figure $2 \mathrm{~g}$ ), so we can conclude that there was very little, if any expression from the mutant paternal allele.

\subsection{Lack of Methylation at the Nesp DMR Is Due to Truncation of Nespas}

To determine whether the lack of methylation at the Nesp DMR in +/Nespas- $T^{\text {int2 }}$ was due to truncation of Nespas or due to the physical disruption of the Nesp locus, we analysed +/Nesp ${ }^{\text {trun }}$ mutant mice [19] which had the polyadenylation cassette inserted at the same location as in Nespas- $T^{\text {int } 2}$, but in the opposite orientation so that truncation of Nespas should not occur (Figure 3a). The Nesp ${ }^{\text {trun }}$ allele was originally generated to truncate maternal Nesp as a functional test for the role of Nesp transcription [19], but the level of Nespas and the methylation status at the Nesp DMR on paternal inheritance were not analysed.

Here, we show that, on paternal inheritance, the insertion in Nesp $p^{\text {trun }}$ had no effect on the level of Nespas (Figure 3b) and that full expression of Nespas from the paternal allele was associated with methylation of the Nesp DMR in cis (Figure 3c). As expected from the methylation analysis, there was no significant difference in the levels of Nesp, Gnasxl, or Exon1A in wild-type and +/Nesp trun (Figure 3d). Thus, our data indicate that the lack of methylation at the Nesp DMR in +/Nespas- ${ }^{\text {int2 }}$ is due to truncation of Nespas rather than physical disruption of the locus. Thus, methylation of Nesp on the paternal allele is controlled by the Nespas non-coding macro RNA gene that is transcribed from a methylation sensitive promoter within the ICR of the Gnas cluster.

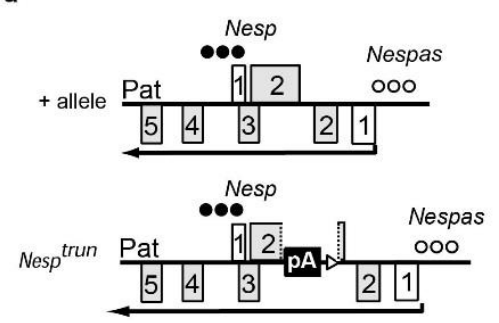

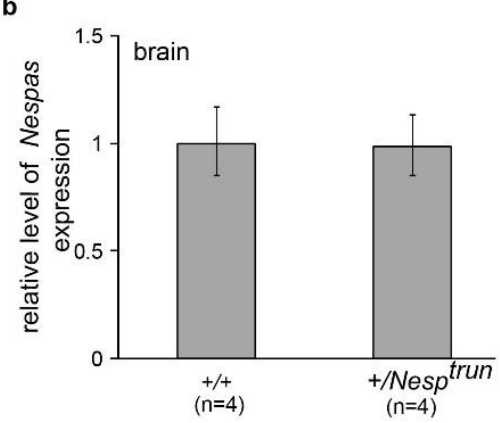

Figure 3. Cont. 

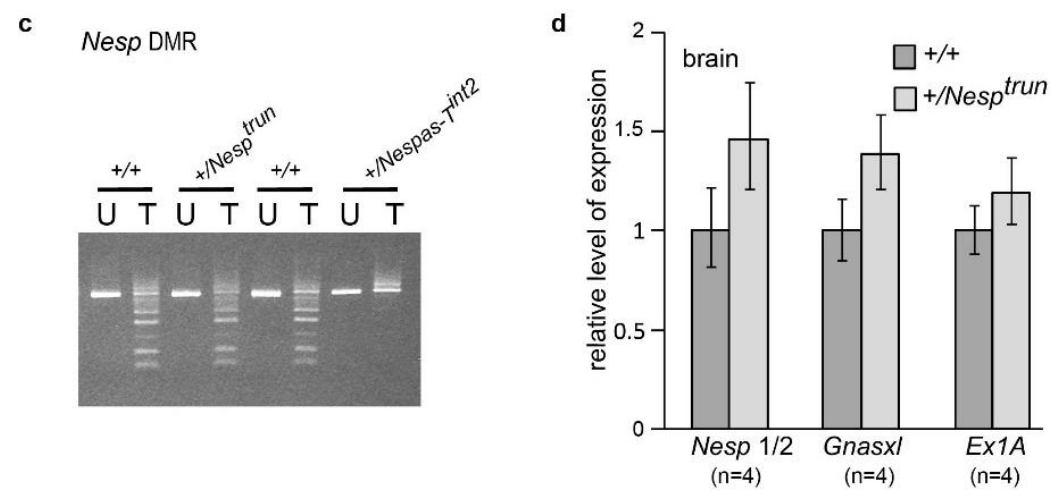

Figure 3. Transcription and methylation status of the Gnas cluster is unaltered on paternal inheritance of Nesp ${ }^{\text {trun }}$. (a) Schematic summary of the transcriptional and methylation status of Nesp and Nespas on the paternal allele in wild-type and +/Nesp $p^{\text {trun }}$. The pA insertion will truncate Nesp if expressed. Row of filled circles, methylated allele; row of unfilled circles, unmethylated allele; (b) The level of Nespas exon 1 spliced onto exon 2 is unaltered in +/Nesp $p^{\text {trun; }}$ (c) Methylation at the Nesp DMR is unaffected. PCR products from bisulphite-treated DNAs were separated on 3\% agarose gels as either undigested (U) or digested with Taq1 (T). Digestion products in the $\mathrm{T}$ lanes represent methylation of the $\mathrm{CpG}$ dinucleotide in the Taq1 recognition sequence. Genomic DNA from +/Nespas- $T^{\text {int } 2}$ in which both alleles were unmethylated was included as a negative control; (d) The levels of Nesp, Gnasxl and ExonlA (ExlA) are unaffected.

\subsection{Truncation of Nespas after the Nesp Promoter}

To narrow down the region of Nespas responsible for silencing, we inserted the polyadenylation cassette from the rabbit $\beta$-globin gene into Nespas intron 4 in an orientation (Ap) to truncate Nespas $12.6 \mathrm{~kb}$ from its promoter and upstream of the Nesp promoter (nucleotides 139,675-140,530; defined in Figure S1). The gene targeting details are shown in Figure 4a,b, and we designated the new allele Nespas- $T^{\text {int } 3}$.

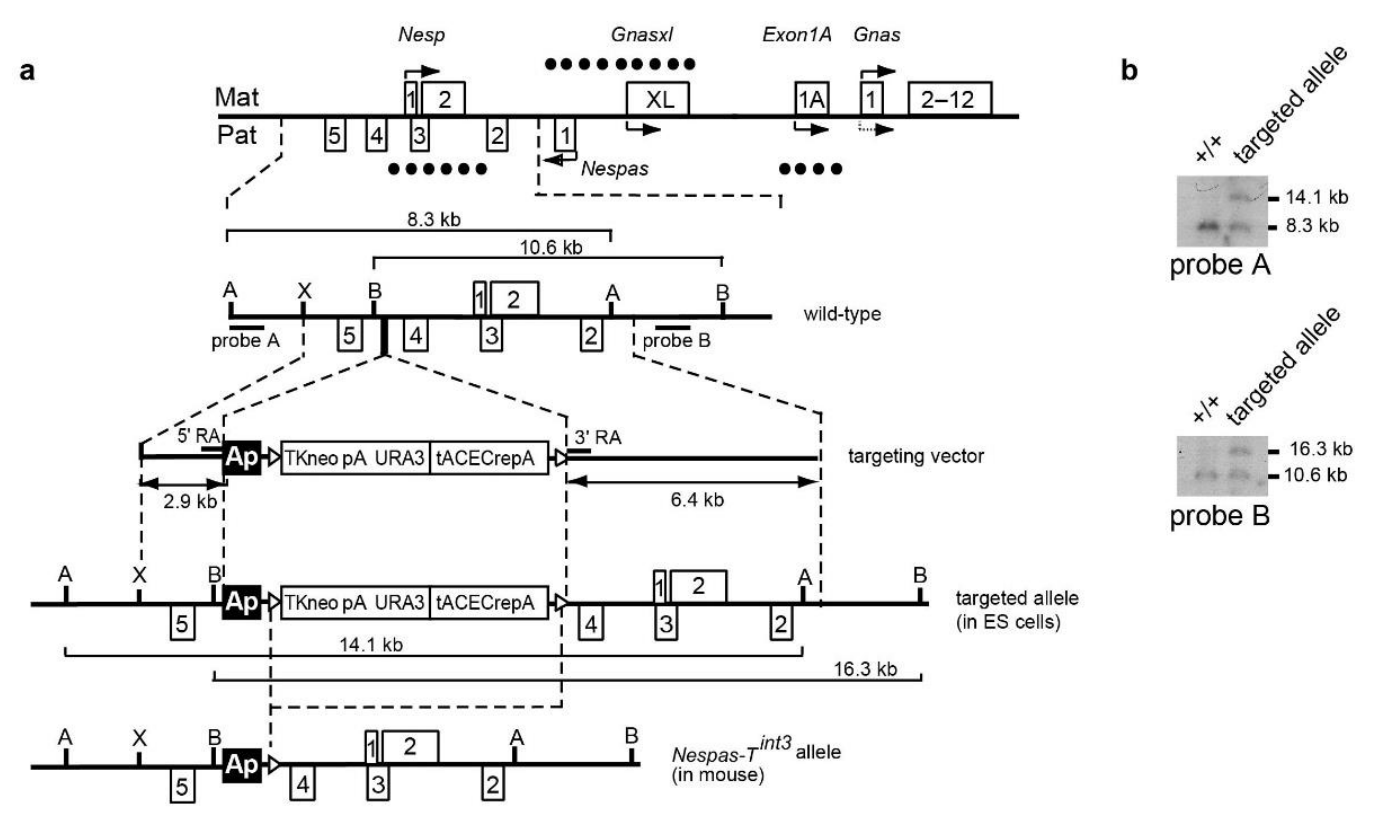

Figure 4. Cont. 
C



g

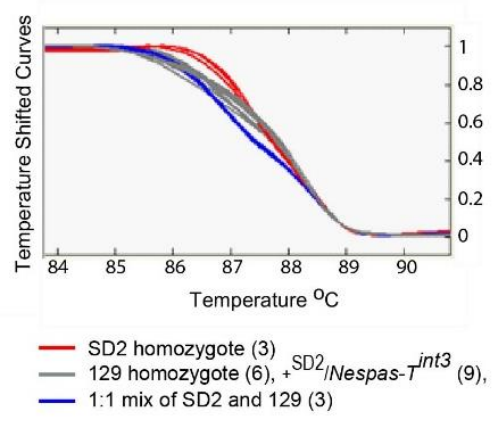

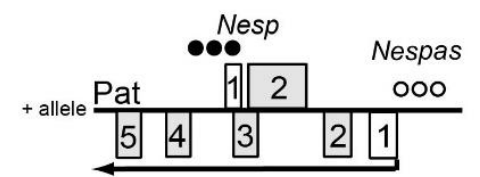

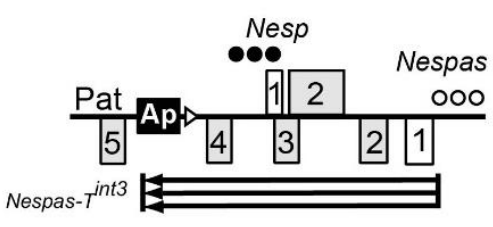

h Nesp DMR $+\mathrm{SD} 2 /+$


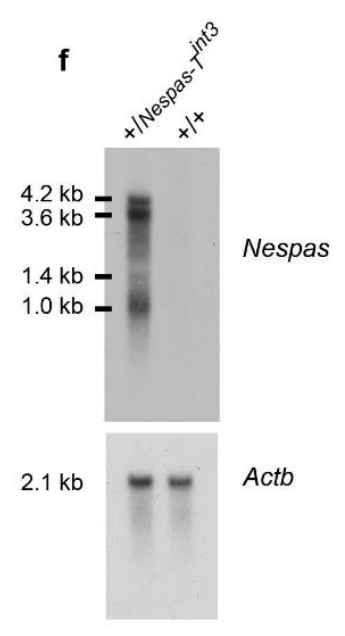

i

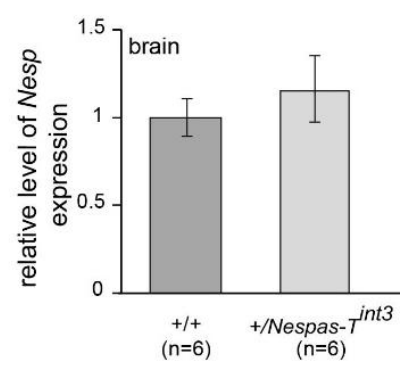

Figure 4. Truncation of Nespas after the Nesp promoter in Nespas intron 4. (a) Schematic of the mouse Gnas locus showing the site of insertion of the rabbit $\beta$-globin polyadenylation cassette (black box labelled Ap) to truncate Nespas and the position of the selection cassette flanked by loxP sites (open triangles). The selection cassette was deleted on germline transmission and the targeted allele was designated Nespas- $T^{\text {int3 }}$. A, AflII; B, BsrGI; X, XhoI; (b) Southern analysis of ES cell DNA showing correct targeting. Correctly targeted clones were identified by the presence of a $14.1 \mathrm{~kb}$ AflII fragment detected with the $5^{\prime}$ external probe, probe A. Correct targeting at the $3^{\prime}$ end was confirmed by the detection of a $16.3 \mathrm{~kb}$ BsrGI fragment with probe B; (c) Nespas is downregulated after the insertion; (d) Nespas is upregulated before the insertion. There was a greater fold difference in the spliced form (exon 1 spliced onto exon 2) than the unspliced form (intron 4); (e) Nespas is truncated and abundant in +/Nespas- $T^{\text {int3 }}$; (f) Northern blot assay of Nespas and loading control Actb; (g) Light Scanner melt profile, using a SNP in Nespas exon 3, to show Nespas is expressed from the paternal allele in $+/$ Nespas- $T^{\text {int3 }}$; (h) Bisulphite analysis showing the Nesp DMR is unmethylated on the maternal allele and methylated on the paternal allele in $+{ }^{\mathrm{SD} 2} / N e s p a s T^{\text {int3 }}$;

(i) The Nesp level is unaffected in +/Nespas- $T^{\text {int3 }}$.

The mutant allele Nespas- $T^{\text {int } 3}$ should give rise to a Nespas transcript that spans the Nesp promoter. Following paternal transmission we determined whether Nespas was truncated by Taqman RT-qPCR analysis. An assay immediately $3^{\prime}$ of the insertion with respect to Nespas transcription, specific to Nespas intron 4 and upstream of the Nesp promoter, detected a 75\% reduction in Nespas level in +/Nespas- $T^{\text {int } 3}$ relative to wild-type ( $n=6 ; P=8.2 \times 10^{-6}$; Figure $4 \mathrm{c}$ ). In contrast, an assay $5^{\prime}$ of the insertion with 
respect to Nespas transcription, specific for Nespas exon 1 spliced onto exon 2 showed an 87-fold increase in Nespas compared to wild-type littermates in $+/$ Nespas $-T^{\text {int3 }}$ on a $129 \mathrm{~S} 9 / \mathrm{SvEvH}$ background generated from one clone $\left(n=5, P=2.1 \times 10^{-11}\right.$; Figure $\left.4 \mathrm{~d}\right)$, a 59-fold increase in +/Nespas- $T^{\text {int } 3}$ generated from a second independently targeted clone $\left(n=6, P=3.0 \times 10^{-4}\right)$ and an 80 -fold increase in $+/$ Nespas $-T^{\text {int3 }}$ on a SD2 background $\left(n=6 ; P=5.9 \times 10^{-11}\right)$. Thus, the increase in Nespas in two independently targeted clones suggests the effect was due to the insertion rather than a non-specific abnormality in the ES cells. Furthermore, as Nespas was truncated upstream of Nesp in +/Nespas- $T^{\text {int } 3 \text {, }}$ an RT-qPCR assay specific for the truncated unspliced form of Nespas could be carried out. An RTqPCR Taqman assay in Nespas intron 4, 5' of the insertion with respect to Nespas transcription, and upstream of the Nesp promoter (Table S2) showed that the unspliced form of Nespas was raised 17-fold when compared with the wild-type level ( $n=5 ; P=5.5 \times 10^{-8}$; Figure $\left.4 \mathrm{~d}\right)$. Once again, the low level of Nespas detected after the insertion compared with the high level detected before the insertion showed that most of the transcripts were truncated as summarised in the schematic in Figure 4e.

The raised expression of Nespas in +/Nespas- $T^{\text {int3 }}$ was verified by RNA blot analysis using a single stranded probe that would detect spliced and unspliced transcripts. The Nespas transcripts which are detected as a smear [20], were highly elevated in newborn brain from +/Nespas- $T^{\text {int3 }}$ compared to wild-type (Figure 4f).

Furthermore, Nespas was shown to be monoallelically expressed from the paternal allele by melt curve analysis (Figure 4g). The melt curves show a decrease in fluorescence as the duplexes melt and the bound LCGreen plus is released into solution. The melt curves for the heteroduplex control (1:1 mix of 129S9/SvEvH and SD2 cDNA; blue line) and for the homoduplex controls 129S9/SvEvH and SD2 (grey line and red line, respectively) were distinct, thus making it possible to distinguish allele-specific Nespas expression. The melt curve for the duplex derived from +/Nespas- $T^{\text {int } 3}$ brain (grey) aligned with the curve for the homoduplex control 129S9/SvEvH which was also grey. Thus, Nespas is expressed from the $129 \mathrm{~S} 9 / \mathrm{SvEvH}$ allele in $+/$ Nespas- $T^{\text {int3 }}$, which is the targeted paternally inherited allele.

\subsection{The Paternal Nesp DMR Is Methylated When Nespas Is Truncated after the Nesp Promoter}

We next investigated the effect of a high level of Nespas RNA truncated after the Nesp promoter on the methylation of the Nesp DMR on the paternal allele in $+/$ Nespas $-T^{\text {int } 3}$. Bisulphite analysis of both the maternal and paternal alleles of one wild-type $\left(+{ }^{\mathrm{SD} 2} /+\right)$ and two mutant $\left(+{ }^{\mathrm{SD} 2} /\right.$ Nespas $\left.-T^{\text {int3 }}\right)$ newborn brains showed no loss of methylation on the paternal allele of $+/ N e s p a s-T^{\text {int3 }}$ compared with that of a wild-type sibling and there was no gain of methylation on the maternal allele (Figure 4h). As expected from the methylation analyses, an RT-qPCR Taqman assay showed there was no difference in the level of Nesp in +/Nespas- $T^{\text {int3 }}$ when compared to the wild-type level (Figure 4i) indicating that Nesp was not expressed from the mutant paternal allele. Furthermore, there was no difference in Nesp level in mice generated from a second independently targeted clone.

Thus, paternally expressed Nespas must span the Nesp promoter in order to methylate the paternal Nesp DMR. Interestingly, even though Nespas was very abundant in +/Nespas- $T^{\text {int } 3}$ the Nesp DMR on the maternal allele remained unmethylated, thus showing that increased Nespas does not force the silencing of Nesp in trans. 


\subsection{Stability of Truncated Nespas $R N A$ in $+/$ Nespas- $T^{\text {int } 3}$}

The increased abundance of Nespas RNA in +/Nespas- $T^{\text {int2 }}$ and +/Nespas- $T^{\text {int3 }}$ compared to wild-type could be due to greater stability of truncated Nespas RNA. The stability of Nespas RNA was analysed in +/Nespas- $T^{\text {int3 }}$ and wildtype sibs by inhibiting total cellular transcription in mouse embryonic fibroblast (MEF) cells using Actinomycin D, a procedure used previously to test the stability of Airn RNA [21].

Myc and Airn were used as controls for unstable RNAs (Figure 5a). Most Myc RNA had disappeared after one hour of exposure, and most Airn and unspliced Nespas RNAs after eight hours exposure, to Actinomycin D. The half-life of Myc RNA was $0.4 \mathrm{~h}$, that of Airn RNA was $2.1 \mathrm{~h}$, identical to that previously found in MEFs [21]. Unspliced Nespas RNA was also found to be unstable with a half-life of $1.6 \mathrm{~h}$. The spliced forms showed increased stability with almost $70 \%$ still present after eight hours exposure (Figure 5a), and we were unable to obtain a half-life for the spliced forms under the experimental conditions used. The mutant truncated unspliced Nespas RNA in +/Nespas- $T^{\text {int3 }}$ with a half-life of $18.6 \mathrm{~h}$ was over 10 times as stable as wild-type (Figure $5 \mathrm{~b}$ ).
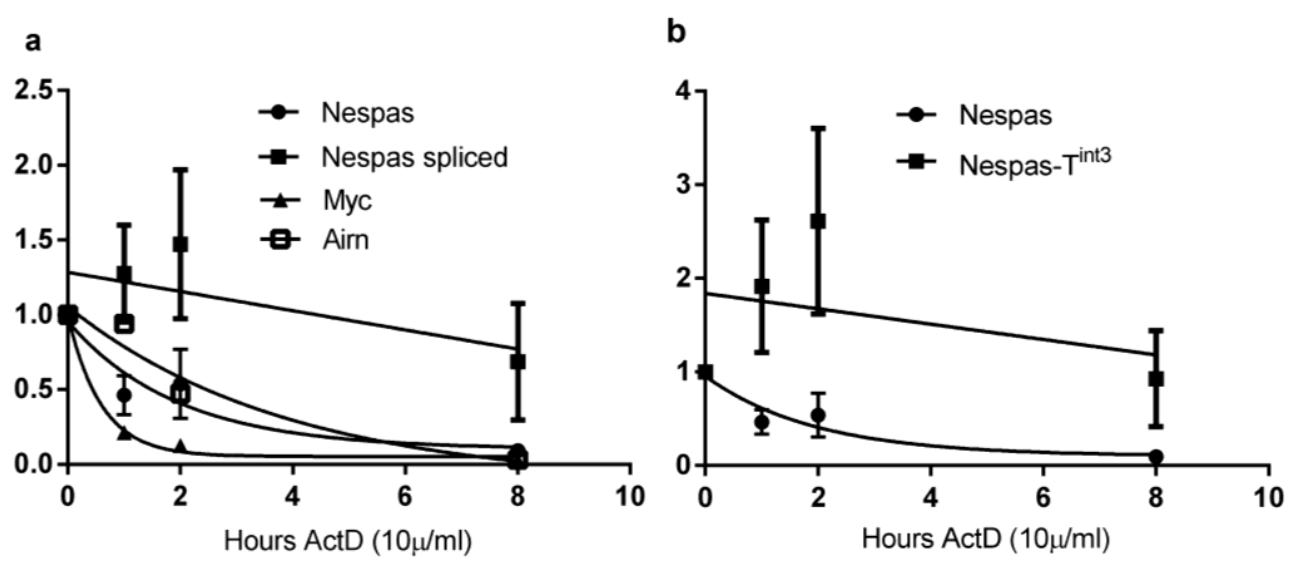

Figure 5. Stability of Nespas RNA (a) Wild-type Nespas RNA. Mouse embryonic fibroblasts MEFs were treated with ActinomycinD $(10 \mu \mathrm{g} / \mathrm{mL})$. Control untreated samples were set to $100 \%$ and Actinomycin D treated samples are shown as a $\%$ of controls. A onephase exponential decay curve was constructed by Prism4 for unspliced Nespas RNA (black circle), spliced Nespas RNA (black square), Myc RNA (black triangle) and Airn RNA (open square); (b) Stability of Nespas-T $T^{\text {int3 }}$ RNA. Wild-type Nespas RNA (black circle) and Nespas- $T^{\text {int3 }}$ RNA (black square). 


\subsection{Reduced Level of Gnasxl on Paternal Transmission of Nespas- $T^{\text {int2 }}$}

The level of Gnasxl was reduced by $60 \%$ in +/Nespas- $T^{\text {int } 2}$ when compared with wild-type $(n=6$, $P=3.8 \times 10^{-4}$, Figure 6a). Although $+/$ Nespas- $T^{\text {int2 }}$ appeared to be normal at birth, were generated at the expected Mendelian frequency (49.6\% of 244 newborns) and showed normal viability thereafter, within a few days they were seen to be thinner than their wild type litter mates and by postnatal day 14 weighed $67.3 \%$ of their wild-type litter mates $\left(P=8.35 \times 10^{-11} ;+/+n=27,+/\right.$ Nespas $\left.-T^{\text {int } 2} n=23\right)$. Growth retardation is typical of mutants with reduction in Gnasxl levels [8,15,22-24]. Expression of Exon1A and Gnas was found to be unaffected (Figure 6a,b).

+/Nespas- $T^{\text {int3 }}$ mice were generated at the expected Mendelian frequency at birth $(45.6 \%$ of 171 newborns) and showed normal viability thereafter. No adverse phenotype was observed which is in keeping with the normal expression of Gnasxl, Exon1A, and Gnas found in these mice (Figure 6c,d).

a

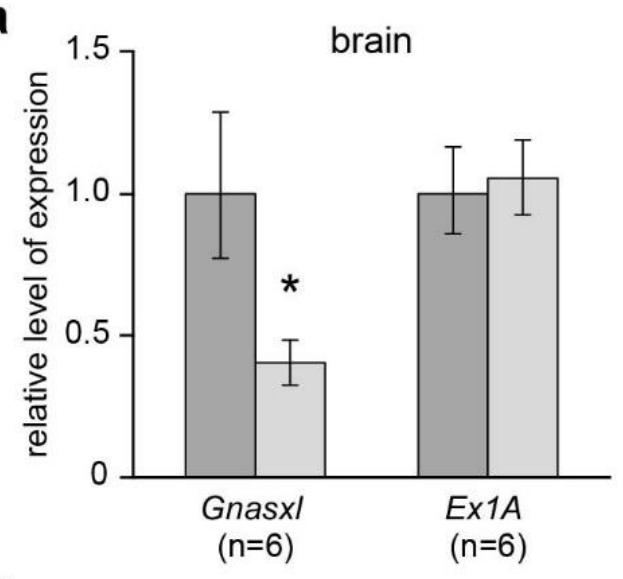

b

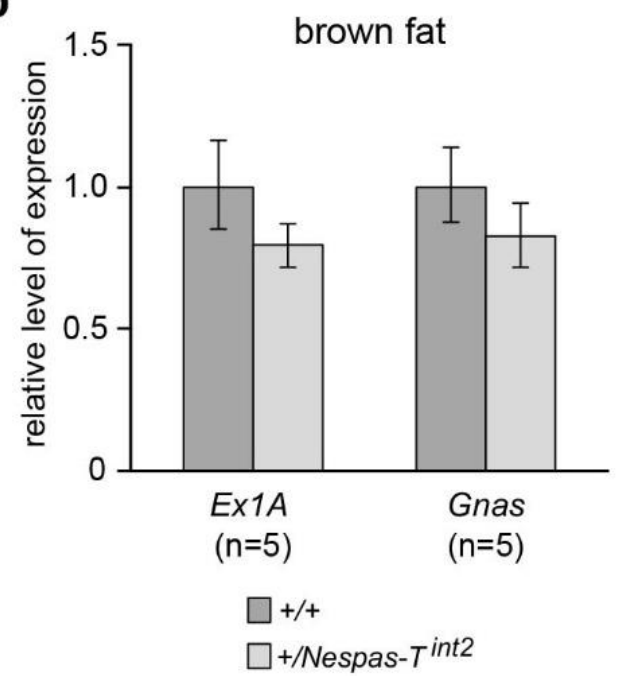

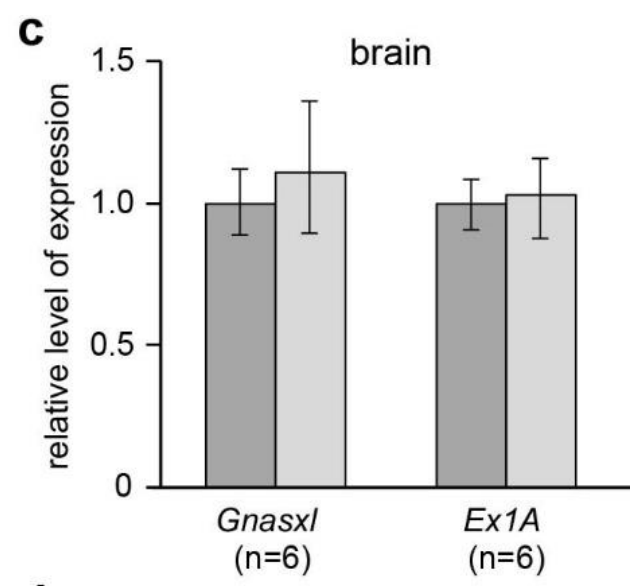

d

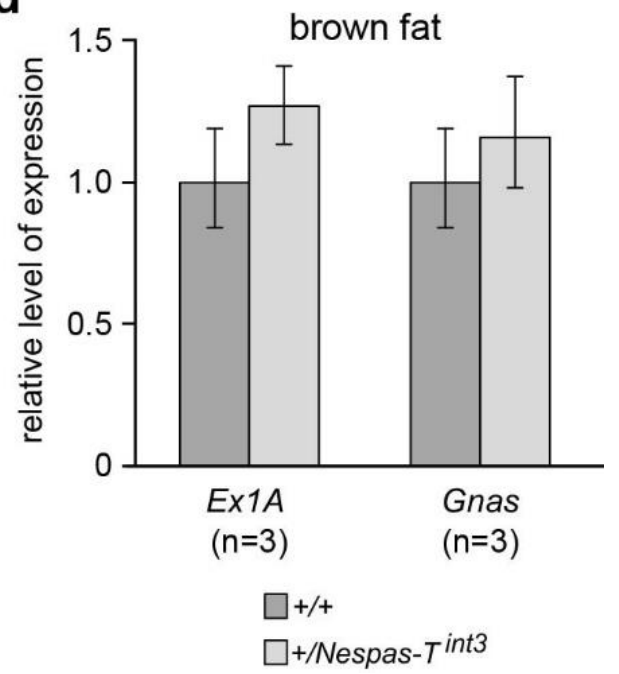

Figure 6. Levels of Gnasxl, Exon1A and Gnas in +/Nespas- $T^{\text {int2 }}$ and $+/$ Nespas $-T^{\text {int3 }}$. (a) and (b) Gnasxl is downregulated in +/Nespas-T ${ }^{\text {int } 2}$ whereas Exon1A (Ex1A) and Gnas are unaffected; (c) and (d) Gnasxl, ExonlA and Gnas are unaffected in +/Nespas-T $T^{\text {int3 }}$. Gnasxl and ExlA levels were measured in newborn brain whereas Gnas and ExlA were analysed in brown fat, a tissue in which Gnas is predominantly expressed from the maternal allele [25,26]. 


\section{Discussion}

From previous work, it was known that methylation of the Nesp DMR correlates with silencing of Nesp expression [4,11]. We have shown here that methylation of the Nesp DMR is dependent on transcription of Nespas across a $2.6 \mathrm{~kb}$ region that includes the Nesp promoter. In the present study, we see truncation of most but not all Nespas transcripts in Nespas- $T^{\text {int } 2}$; around 20\% cross the Nesp DMR, but this weak Nespas expression was insufficient for methylation of the DMR. This result extends earlier findings that the DMR was unmethylated when Nespas was very weakly expressed [4]. Thus, Nespas resembles Airn, [2] the well studied antisense long non-coding RNA at the $I g f 2 r$ cluster, in that both run through, and are required for methylation of the promoters of their respective sense counterparts, Nesp and Igf $2 r$. Furthermore, when Nespas and Airn [27] are weakly expressed Nesp and Igf $2 r$ do not acquire methylation.

Methylation of the Nesp DMR occurs between $3.5 \mathrm{dpc}$ and $8.5 \mathrm{dpc}$ [15,28]. Nesp is not robustly expressed until $6.5 \mathrm{dpc}$ [24,29], but Nespas is expressed much earlier, from the two cell stage onwards [29]. Expression of Nespas is associated with elevated levels of histones H3K36me3 and unmethylated H3K4 at the Nesp promoter [4]. H3K36me3 and unmethylated H3K4 are permissive for DNA methylation [30-32], and so it is feasible that transcription of Nespas through the Nesp promoter region enables DNA methylation through the deposition of histone marks permissive for DNA methylation. Whether DNA methylation of the Nesp promoter is instrumental in initiating silencing of Nesp is not known and DNA methylation may be required to stabilise silencing of Nesp once silencing is underway.

Methylation of the Nesp DMR may also be required to maintain full expression of Gnasxl. Nespas- $T^{\text {int2 }}$ is the fourth mutant in which the Nesp DMR is unmethylated on paternal inheritance and Gnasxl is downregulated. Unlike Nespas- $T^{\text {int } 2}$, there is expression of Nesp from the mutant paternal allele in the three other mutants, $\triangle N A S-D M R$, Nespas- $T^{\text {exl }}$ and Nesp- $T^{\text {int }}[4,11,24]$. Furthermore, Gnasxl expression is reduced even though the Gnasxl DMR is unmethylated [4,11]. These findings are consistent with presence of a silencer for Gnasxl within the Nesp DMR that is active when the Nesp DMR is unmethylated and inactive when the Nesp DMR is methylated. Deletion of the paternal methylated Nesp DMR does not affect expression of Gnasxl [25] which accords with the prediction of a silencer. We propose that DNA methylation at the Nesp DMR is needed to inactivate a silencer of Gnasxl.

Although the paternal Nesp DMR was unmethylated in Nespas- $T^{\text {int } 2}$, the expression of Nesp was not detectably affected and Nesp remained silent. It was known that very low levels of Nespas can downregulate Nesp [4] and therefore in Nespas- $T^{\text {int } 2}$ it is likely that the $20 \%$ or so of wild-type Nespas transcripts that are not truncated and cross the Nesp DMR are sufficient to silence Nesp. However, we cannot exclude the possibility that transcription of Nesp in Nespas- $T^{\text {int2 }}$ was diminished by the close proximity of the insertion to the Nesp promoter. Taken together with findings that total loss of Nespas expression across the Nesp promoter results in complete de-repression of paternal Nesp $[4,11]$ it can be concluded that expression of Nespas is definitely required for silencing of Nesp. Thus, although silencing the Nesp promoter only requires weak expression of Nespas, higher levels of Nespas are required for DNA methylation of the silenced region.

It is not known whether it is transcription of Nespas or the Nespas transcript that is instrumental is silencing Nesp. Given the sense-antisense overlap Nespas may downregulate Nesp by a transcription based mechanism as reported for the silencing of Igf2r by Airn [33], or by a transcript based RNA 
interference mechanism. Even when transcript levels were highly elevated Nespas acted exclusively in cis, hinting that transcription of Nespas through the Nesp promoter rather than the Nespas transcript itself may result in silencing of Nesp. However, it has been reported that Nespas RNA is associated with the chromatin modifying Polycomb repressive complex 2, so a silencing role for the Nespas transcript cannot be excluded [34].

We show here that wild-type unspliced Nespas ncRNA is unstable, with the lack of stability resembling that found for Airn and Tsix ncRNAs [21,35]. As found with Airn, the spliced forms of Nespas are more stable than the unspliced form which may indicate that unspliced Nespas RNA is rapidly degraded. Unlike the long non coding antisense Airn and Kcnqlot1 RNAs whose expression levels are unaltered when truncated [2,3,33], truncation of Nespas RNA results in very high increase in expression levels. Truncated Nespas RNA is much more stable than the wild-type form, and this increased stability may well account in large part for the increased expression levels of truncated Nespas.

There is mounting evidence that the role of Nespas is to suppress expression of Nesp and induce methylation of the Nesp DMR on the paternal chromosome, thereby indirectly controlling paternal expression of protein coding transcripts Gnasxl and Gnas. On the paternal chromosome in wild-type, Nesp is repressed, the Nesp DMR is methylated, Gnasxl is fully expressed and Gnas is suppressed tissue specifically (Figures 1 and 7). However, whereas methylation of the Nesp DMR appears to be crucial for full paternal Gnasxl expression, it is suppression of Nesp transcription that is essential for tissue specific paternal repression of Gnas. Ectopic paternal expression of Nesp results in loss of imprinting of Gnas [24]. This occurs because ectopic transcription of Nesp results in methylation of the Exon1A DMR; the Exon1A DMR controls the imprinted expression of Gnas [25,28] and methylation of the Exon1A DMR results in upregulation of Gnas in tissues where Gnas shows imprinted expression [4,11,24]. Thus, at the Gnas cluster, Nespas expression is key to defining paternal gene expression. The primary role of Nespas may be to repress Nesp expression and induce methylation of the Nesp DMR in order that appropriate levels of Gnas and Gnasxl are maintained on the paternal allele.
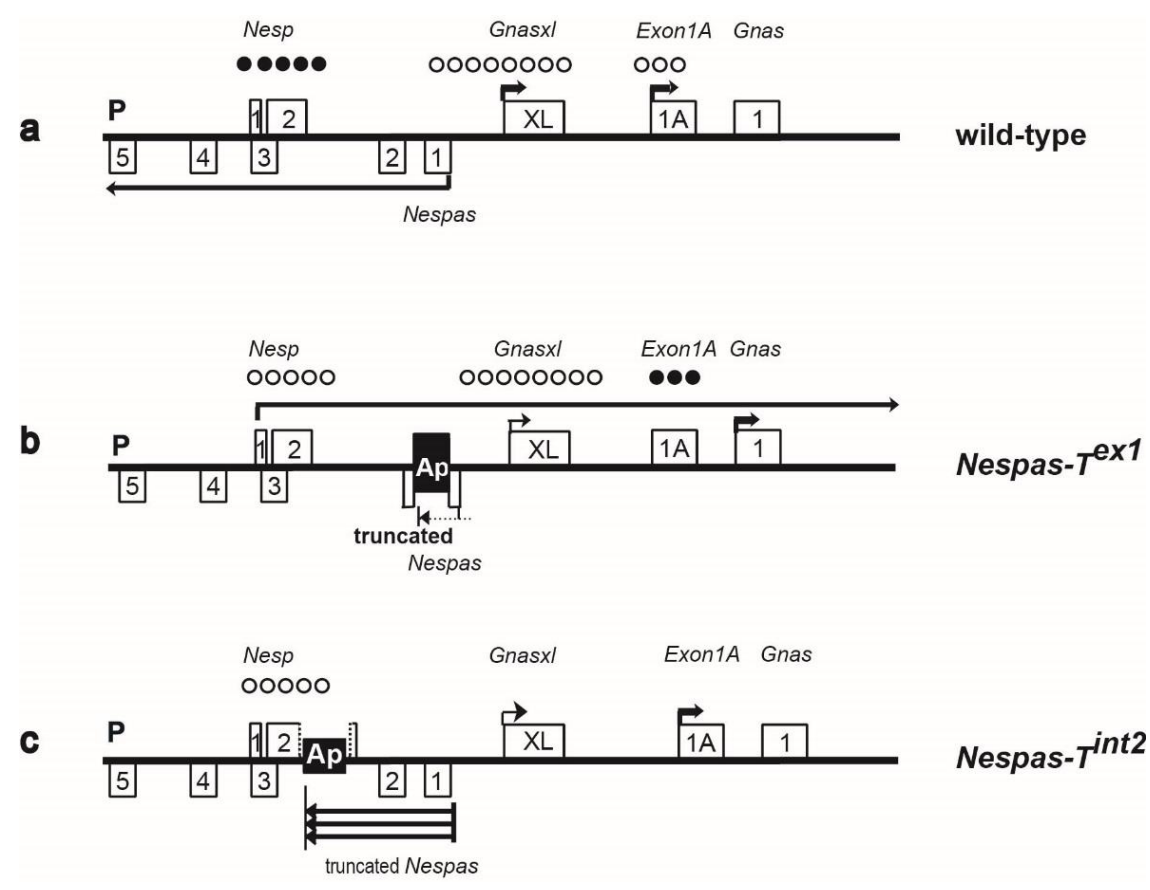

Figure 7. Cont. 


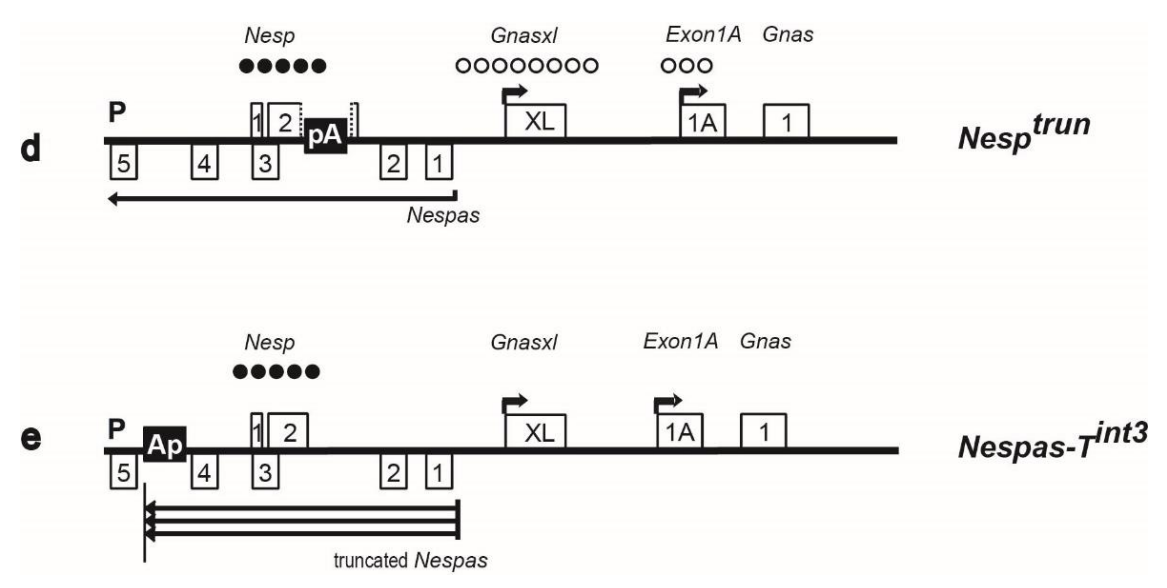

Figure 7. Summary of the transcriptional and methylation status of the paternal allele of the imprinted Gnas cluster in wild-type and mutant mice. (a) wild-type; (b) +/Nespas- $T^{\text {exl }}$; (c) $+/$ Nespas- $T^{\text {int } 2}$; (d) +/Nesp ${ }^{\text {trun }}$; (e) $+/$ Nespas- $T^{\text {int3 }}$. The Ap insertion truncates Nespas and the pA insertion will truncate Nesp if expressed. The approximate positions of the differentially methylated regions (DMRs) are shown by rows of filled circles on the methylated allele and unfilled circles on the unmethylated allele. Arrows show the start and direction of transcription, with thin arrows indicating weak transcription. Multiple arrows in (c) and (e) show increased levels of truncated Nespas. Exons 2-12 of Gnas are not shown. The figure is not drawn to scale. Information for (b), +/Nespas- $T^{\text {exl }}$, taken from [4,24].

\section{Material and Methods}

\subsection{GenBank Accession Number}

The nucleotide numbers referred to in this publication correspond to sequence accession number AL593857.10.

\subsection{Generation of Targeted Alleles}

To generate the Nespas- $T^{\text {int2 }}$ allele, a targeting construct was designed to insert a polyadenylation cassette from the rabbit $\beta$-globin gene [2] into intron 2 of Nespas, between nucleotides 141,635-141,636, in an orientation (designated Ap) that would be expected to truncate Nespas (Figure 2a). The construct was generated by homologous recombination in yeast as described previously [4] except that pRAY1 vector [36] was used. The targeting construct was linearised with NotI and electroporated into R1 mouse ES cells [37]. Colonies surviving G418 selection were screened by Southern blot analysis of AflII digested DNA probed with genomic fragment probe A (nucleotides 134,606-136,087) that lies 5' of the targeting construct arm. XhoI digested DNA was probed with probe B genomic fragment (nucleotides 147,539-148,139) that lies 3' of the targeting construct arm (Figure 2a,b). Targeted ES cells were karyotyped, and there was no evidence of chromosomal changes [38]. Chimeras were generated by injecting two correctly targeted ES clones into C57BL/6J blastocysts. Germline transmission of the targeted allele was confirmed by PCR using primers in Table S1. Transmitting male chimeras were crossed with $\mathrm{Tg} / \mathrm{ACTB}$-cre/2Mrt/J females that were heterozygous for $\beta$-actin-Cre, a transgene ubiquitously expressed in embryos at or before the blastocyst stage [39,40]. Offspring were genotyped 
for the Nespas- $T^{\text {int2 }}$ and $\beta$-actin-Cre alleles and double heterozygotes were crossed with 129S9/SvEvH mice. Nespas- $T^{\text {int2 }}$ carriers were maintained on a $129 \mathrm{~S} 9 / \mathrm{SvEvH}$ background. Excision of the selection cassette was confirmed by PCR amplification across the remaining loxP site. The sequence of the primers for detecting the Nespas- $T^{\text {int2 }}$ allele and $\beta$-actin-Cre allele are provided in Table S1. Nesp ${ }^{\text {trun }}$ carriers had previously been generated and were maintained on a C57BL/6 background [19].

The Nespas- $T^{\text {int } 3}$ allele was generated by using a targeting construct designed to insert the polyadenylation cassette from the rabbit $\beta$-globin gene into intron 4 of Nespas, between nucleotides 139,024 and 139,025 and upstream of the Nesp promoter in an orientation (designated Ap) that would be expected to truncate Nespas (Figure 4a). The construct was generated by homologous recombination in yeast as described above using pRAY-Cre vector [23] that allows self-excision of the selection cassette upon germline transmission. As described above for the generation of the Nespas- $T^{\text {int } 2}$ allele, the targeting construct was linearised with NotI and electroporated into R1 mouse ES cells [37]. Correctly targeted clones were identified by Southern blot analysis of $A f l I I$ digested DNA, probed with genomic fragment probe A described above. BsrGI digested DNA was probed with genomic fragment probe B described above (Figure 4a,b). Chimeras were generated by injecting two correctly targeted ES clones into C57BL/6J blastocysts. Germline transmission of the targeted allele was confirmed by PCR using primers in Table S1. Nespas- $T^{\text {int3 }}$ carriers were maintained on a 129S9/SvEvH background.

All mouse studies were conducted under guidance issued by the Medical Research Council in "Responsibility in the Use of Animals in Bioscience Research" (May 2008) and under the authority of Home Office Project Licence Number 30/2526 and the approval of the Harwell Ethics Committee. For the characterisation of +/Nespas- $T^{\text {int2 }}$ and $+/$ Nespas $-T^{\text {int } 3}$, mice were examined daily and observations recorded using a numerical system on a welfare scoring sheet. From birth onwards, animals were scored for up to 12 parameters affecting feeding, growth, morphology and activity. Mice were housed in Tecniplast IVC 1284L caging with a maximum number of five mice per cage. All cages contained pine bedding (Datesand grade 6) and Datesand rodent tunnels and shredded paper for environmental enrichment. All mice had free access to water and diet (Special diet services (Dietex, Witham, UK) RM3 (E)) in a 12-h light-dark cycle with room temperature $19-22{ }^{\circ} \mathrm{C}$.

\subsection{Genotyping Assay}

PCR quality mouse genomic DNA was prepared from ear/toe clip biopsies by a modified alkaline lysis protocol called HotSHOT [41]. Briefly the biopsies were incubated in $100 \mu \mathrm{L}$ of $50 \mathrm{mM} \mathrm{NaOH}$ for $1 \mathrm{~h}$ at $95{ }^{\circ} \mathrm{C}$, then neutralised by adding $10 \mu \mathrm{L}$ of $1 \mathrm{M}$ Tris- $\mathrm{HCl}(\mathrm{pH}$ 8) followed by dilution with $100 \mu \mathrm{L}$ of water. One microlitre of the final volume was added to Reddy Mix PCR Master Mix (Thermo Scientific, Waltham, MA, USA) containing the appropriate primers for PCR amplification.

\subsection{Mouse Weight Data Analysis}

Individual mice were weighed at 14 days. The weight comparisons of mutant mice and their normal sibs were assessed by Student's $t$-test on the basis of weight ratios within litters as this eliminates weight differences between different litters attributable to varying litter sizes. 


\subsection{Expression Analyses}

RNA extraction and reverse transcription-quantitative real-time PCR (RT-qPCR) was performed as described previously [4]. Transcript levels were measured in newborn tissues. Nesp, Nespas and Gnasxl levels were assayed in brain, Exon1A in brain and brown fat, and Gnas in brown fat. For analysis of Nespas in +/Nespas- $T^{\text {int2 }}$ and +/Nespas- $T^{\text {int } 3}$ Taqman assays for the RT-qPCR were designed 3' and 5' of the insertions with respect to Nespas transcription. For $+/$ Nespas $-T^{\text {int } 2}$ the $3^{\prime}$ assay was Nespas intron 4 (AIGJPKJ; Table S2) and the 5' assay was Nespas ex1/ex2 (Mm01248137.m1). For +/Nespas-T $T^{\text {int3 }}$, the 3' assay was Nespas intron 4 (AI88XP8) [4], the 5' assay for spliced Nespas was Nespas ex1/ex2 (Mm01248137.m1) and the 5' assay for unspliced Nespas was Nespas intron 4 (AIGJPKJ). Nespas was analysed in $+/$ Nesp $^{\text {trun }}$ using assay Nespas ex1/ex2 (Mm01248137.m1). For analysis of Nesp in +/Nespas- $T^{\text {int2 }}$ and +/Nesp ${ }^{\text {trun }}$ Taqman assay Nesp ex1/ex2 (nesp0-N0) was used. Nesp was measured in +/Nespas $-T^{\text {int-3 }}$ using Taqman assay Nesp ex2/ex3 (AIX020D, Table S2, Note: exon 3 of Nesp is also exon 2 of Gnas). Other assays were for Gnasxl (Mm01717466_g1), Ex1A (Mm01248152_m1), and Gnas (Mm00507037_m1). Samples were analysed in triplicate, and calculations performed using the comparative $\mathrm{C}_{\mathrm{T}}$ method. The values were normalised to the endogenous reference gene, Gapdh (Mm99999915_g1) and the transcript levels were presented as fold change relative to the wild-type sample in relative quantification (RQ) units. Error bars indicate the calculated maximum (RQMax) and minimum ( $\left.R Q_{M i n}\right)$ expression levels, with a $95 \%$ confidence level. Statistical significance was tested using two-sample equal variance, two-tailed distribution Student's $t$-test and is also represented as nonoverlapping error bars if the samples are significantly different $(p<0.05)$.

Poly $(\mathrm{A})^{+}$RNA for blot analysis was extracted using a FastTrack kit (Invitrogen, Carlsbad, CA, USA). Northerns were carried out as previously described [11].

\subsection{PCR Product Melting Curve Analysis for Differentiating SNP Based Allelic Expression}

Melting curve analysis [42] was performed to determine the parental origin of Nespas transcription. Briefly the 90 bp cDNA-derived PCR products, spanning the Nespas exon 3 SNP (nucleotide 140,755; $\mathrm{C}$ in 129S9/SvEvH and T in SD2) were amplified, in the presence of the double-stranded DNA binding dye LCGreen plus, HotShot Mastermix, and $0.2 \mathrm{mM}$ forward and reverse primers (0.2 $\mathrm{mM}$; Table S2). PCR conditions were: $95{ }^{\circ} \mathrm{C}$ for $2 \mathrm{~min}$, then 44 cycles of $95{ }^{\circ} \mathrm{C}$ for $30 \mathrm{~s}, 60{ }^{\circ} \mathrm{C}$ for $30 \mathrm{~s}, 72{ }^{\circ} \mathrm{C}$ for $30 \mathrm{~s}$. Following PCR, the products were heated to $95^{\circ} \mathrm{C}$ and then rapidly cooled to $15^{\circ} \mathrm{C}$ to generate duplexes. The duplexes were melted and the melt curves were obtained using an Idaho Technology LightScanner (Salt Lake City, UT, USA). The decrease in fluorescence was a measure of the bound LCGreen plus being released into solution as the duplex melted. To distinguish allele-specific expression, control cDNA was included to ensure distinct melting curves were obtained for the 129S9/SvEvH and SD2 homoduplexes and heteroduplexes. For the latter, a 1:1 mix of 129S9/SvEvH and SD2 cDNA was used. Each sample was analysed in triplicate.

\subsection{Methylation Analysis}

Methylation at the Nesp DMR was analysed by bisulphite analysis. Briefly genomic DNA, extracted from newborn brain using the Allprep kit (Qiagen Ltd. United Kingdom, Manchester, UK), was treated 
using the EpiTect Bisulfite Kit (Qiagen) and the DNA was amplified using Nesp DMR primers [4] then cloned and sequenced. The parental alleles were distinguished using a variant between 129S9/SvEvH and SD2, at nucleotide 140,755, in Nesp intron 1. Gel analysis of methylation was performed by digestion of the PCR amplified bisulphite-treated DNA with TaqI (recognition sequence TCGA). Digestion products represented methylation of the starting DNA.

\subsection{Luciferase Reporter Assay to Measure Promoter Activity}

Reporter constructs were generated and the assay was done as previously described [25]. The reporter constructs were generated by amplifying the 5' end of the Nesp DMR (nucleotides 139,675-140,530) [14] from mouse PAC clone 583L07 (RPCI21 library) and the product was cloned in both orientations in the BglII site of the pGL3-Basic vector and pGL3-Enhancer vector (Promega UK, Southampton, UK). The plasmids were transfected into HeLa cells and the pGL3-Control and pGL3-Basic vectors were included as positive and negative controls, respectively. The experiment was done twice whereby for each experiment the construct was tested in duplicate and duplicate readings were taken for each sample.

\subsection{Transcription Inhibitor Assay}

Actinomycin D treatment was performed as previously described [21]. Briefly MEFs were prepared from $13.5 \mathrm{dpc}$ embryos and were used at P14-P18. The cells were cultured for $42 \mathrm{~h}$. At time point 0 , the media were removed and the cells were washed with PBS and incubated with media supplemented with $10 \mu \mathrm{g} / \mathrm{mL}$ Actinomycin D (Source BioScience, Nottingham, UK) dissolved in DMSO. Control dishes were incubated with media plus DMSO. At each time point, cells from a control dish and a treated dish were harvested for RNA analysis. Transcript levels were measured by RT-qPCR using Taqman assays Nespas ex1/2 for spliced Nespas RNA, and intron 4 (AIGJPKJ) for unspliced Nespas RNA. The data were taken from three biological replicates, each of which was analysed three times. Each value was normalised to Gapdh.

\section{Acknowledgments}

We thank Denise Barlow and Frank Sleutels for providing the polyadenylation cassette from the rabbit $\beta$-globin gene. We also thank staff of the Mary Lyon Centre for gene targeting and animal husbandry expertise and Adrian Ford for help with the figures. This work was supported by the UK Medical Research Council Awards MC_U142636336 and G0800013, and a Special Committee Studentship from the Biotechnology and Biological Sciences Research Council.

\section{Author Contributions}

C.J.T., C.M.W., J.P. conceived and designed the experiments; C.J.T., C.M.W., S.A.E., S.T.B., S.M., W.T.N. performed the experiments; C.J.T., C.M.W., S.T.B., S.M., W.T.N., J.P. analyzed the data; M.C., S.A.E., M.M.Q., L.T., G.K. contributed new reagents/analytic tools; C.M.W., J.P. wrote the paper.

\section{Conflicts of Interest}

The authors declare no conflict of interest. 


\section{References}

1. Barlow, D.P.; Bartolomei, M.S. Genomic imprinting in mammals. Cold Spring Harb. Perspect. Biol. 2014, 6, doi:10.1101/cshperspect.a018382.

2. Sleutels, F.; Zwart, R.; Barlow, D.P. The non-coding air rna is required for silencing autosomal imprinted genes. Nature 2002, 415, 810-813.

3. Mancini-Dinardo, D.; Steele, S.J.; Levorse, J.M.; Ingram, R.S.; Tilghman, S.M. Elongation of the kcnq1ot 1 transcript is required for genomic imprinting of neighboring genes. Genes Dev. 2006, 20 , 1268-1282.

4. Williamson, C.M.; Ball, S.T.; Dawson, C.; Mehta, S.; Beechey, C.V.; Fray, M.; Teboul, L.; Dear, T.N.; Kelsey, G.; Peters, J. Uncoupling antisense-mediated silencing and DNA methylation in the imprinted gnas cluster. PLoS Genet. 2011, 7, e1001347.

5. Meng, L.; Person, R.E.; Beaudet, A.L. Ube3a-ats is an atypical rna polymerase ii transcript that represses the paternal expression of ube3a. Hum. Mol. Genet. 2012, 21, 3001-3012.

6. Meng, L.; Person, R.E.; Huang, W.; Zhu, P.J.; Costa-Mattioli, M.; Beaudet, A.L. Truncation of ube3a-ats unsilences paternal ube 3 a and ameliorates behavioral defects in the angelman syndrome mouse model. PLoS Genet. 2013, 9, e1004039.

7. Peters, J.; Williamson, C.M. Control of imprinting at the gnas cluster. Epigenetics 2007, 2, 207-213.

8. Eaton, S.A.; Williamson, C.M.; Ball, S.T.; Beechey, C.V.; Moir, L.; Edwards, J.; Teboul, L.; Maconochie, M.; Peters, J. New mutations at the imprinted gnas cluster show gene dosage effects of gsalpha in postnatal growth and implicate xlalphas in bone and fat metabolism but not in suckling. Mol. Cell. Biol. 2012, 32, 1017-1029.

9. Fernandez-Rebollo, E.; Maeda, A.; Reyes, M.; Turan, S.; Frohlich, L.F.; Plagge, A.; Kelsey, G.; Juppner, H.; Bastepe, M. Loss of xlalphas (extra-large alphas) imprinting results in early postnatal hypoglycemia and lethality in a mouse model of pseudohypoparathyroidism ib. Proc. Natl. Acad. Sci. USA 2012, 109, 6638-6643.

10. Ball, S.T.; Kelly, M.L.; Robson, J.E.; Turner, M.D.; Harrison, J.; Jones, L.; Napper, D.; Beechey, C.V.; Hough, T.; Plagge, A.; et al. Gene dosage effects at the imprinted cluster. PLOS ONE 2013, 8, e65639.

11. Williamson, C.M.; Turner, M.D.; Ball, S.T.; Nottingham, W.T.; Glenister, P.; Fray, M.; Tymowska-Lalanne, Z.; Plagge, A.; Powles-Glover, N.; Kelsey, G.; et al. Identification of an imprinting control region affecting the expression of all transcripts in the gnas cluster. Nat. Genet. 2006, 38, 350-355.

12. Williamson, C.M.; Skinner, J.A.; Kelsey, G.; Peters, J. Alternative non-coding splice variants of nespas, an imprinted gene antisense to nesp in the gnas imprinting cluster. Mamm. Genome 2002, 13, 74-79.

13. Peters, J.; Wroe, S.F.; Wells, C.A.; Miller, H.J.; Bodle, D.; Beechey, C.V.; Williamson, C.M.; Kelsey, G. A cluster of oppositely imprinted transcripts at the gnas locus in the distal imprinting region of mouse chromosome 2. Proc. Natl. Acad. Sci. USA 1999, 96, 3830-3835. 
14. Kelsey, G.; Bodle, D.; Miller, H.J.; Beechey, C.V.; Coombes, C.; Peters, J.; Williamson, C.M. Identification of imprinted loci by methylation-sensitive representational difference analysis: Application to mouse distal chromosome 2. Genomics 1999, 62, 129-138.

15. Liu, J.; Yu, S.; Litman, D.; Chen, W.; Weinstein, L.S. Identification of a methylation imprint mark within the mouse gnas locus. Mol. Cell. Biol. 2000, 20, 5808-5817.

16. Holmes, R.; Williamson, C.; Peters, J.; Denny, P.; Wells, C.; Group, R.G.; Members, G.S.L. A comprehensive transcript map of the mouse gnas imprinted complex. Genome Res. 2003, 13, 1410-1415.

17. Robson, J.E.; Eaton, S.A.; Underhill, P.; Williams, D.; Peters, J. Micrornas 296 and 298 are imprinted and part of the gnas/gnas cluster and mir-296 targets ikbke and tmed9. Rna 2012, 18, 135-144.

18. Huang, R.; Barlow, D.P. Personal Communication; CeMM: Vienna, Austria, 2011.

19. Chotalia, M.; Smallwood, S.A.; Ruf, N.; Dawson, C.; Lucifero, D.; Frontera, M.; James, K.; Dean, W.; Kelsey, G. Transcription is required for establishment of germline methylation marks at imprinted genes. Genes Dev. 2009, 23, 105-117.

20. Wroe, S.F.; Kelsey, G.; Skinner, J.A.; Bodle, D.; Ball, S.T.; Beechey, C.V.; Peters, J.; Williamson, C.M. An imprinted transcript, antisense to nesp, adds complexity to the cluster of imprinted genes at the mouse gnas locus. Proc. Natl. Acad. Sci. USA 2000, 97, 3342-3346.

21. Seidl, C.I.; Stricker, S.H.; Barlow, D.P. The imprinted air ncrna is an atypical rnapii transcript that evades splicing and escapes nuclear export. EMBO J. 2006, 25, 3565-3575.

22. Cattanach, B.M.; Peters, J.; Ball, S.; Rasberry, C. Two imprinted gene mutations: Three phenotypes. Hum. Mol. Genet. 2000, 9, 2263-2273.

23. Plagge, A.; Gordon, E.; Dean, W.; Boiani, R.; Cinti, S.; Peters, J.; Kelsey, G. The imprinted signaling protein $\mathrm{xl}$ alpha $\mathrm{s}$ is required for postnatal adaptation to feeding. Nat. Genet. 2004, 36, 818-826.

24. Mehta, S.; Williamson, C.M.; Ball, S.; Tibbit, C.; Beechey, C.; Fray, M.; Peters, J. Transcription driven somatic DNA methylation within the imprinted gnas cluster. PLOS ONE 2015, 10, e0117378.

25. Williamson, C.M.; Ball, S.T.; Nottingham, W.T.; Skinner, J.A.; Plagge, A.; Turner, M.D.; Powles, N.; Hough, T.; Papworth, D.; Fraser, W.D.; et al. A cis-acting control region is required exclusively for the tissue-specific imprinting of gnas. Nat. Genet. 2004, 36, 894-899.

26. Yu, S.; Yu, D.; Lee, E.; Eckhaus, M.; Lee, R.; Corria, Z.; Accili, D.; Westphal, H.; Weinstein, L.S. Variable and tissue-specific hormone resistance in heterotrimeric gs protein alpha-subunit (gsalpha) knockout mice is due to tissue-specific imprinting of the gsalpha gene. Proc. Natl. Acad. Sci. USA 1998, 95, 8715-8720.

27. Stricker, S.H.; Steenpass, L.; Pauler, F.M.; Santoro, F.; Latos, P.A.; Huang, R.; Koerner, M.V.; Sloane, M.A.; Warczok, K.E.; Barlow, D.P. Silencing and transcriptional properties of the imprinted airn ncrna are independent of the endogenous promoter. EMBO J. 2008, 27, 3116-3128.

28. Liu, J.; Chen, M.; Deng, C.; Bourc'his, D.; Nealon, J.G.; Erlichman, B.; Bestor, T.H.; Weinstein, L.S. Identification of the control region for tissue-specific imprinting of the stimulatory g protein alpha-subunit. Proc. Natl. Acad. Sci. USA 2005, 102, 5513-5518.

29. Mehta, S. Imprinting at the Mouse Gnas Cluster; University of Oxford: Oxford, UK, 2012. 
30. Ooi, S.K.; Qiu, C.; Bernstein, E.; Li, K.; Jia, D.; Yang, Z.; Erdjument-Bromage, H.; Tempst, P.; Lin, S.P.; Allis, C.D.; et al. Dnmt3l connects unmethylated lysine 4 of histone h3 to de novo methylation of DNA. Nature 2007, 448, 714-717.

31. Zhang, Y.; Jurkowska, R.; Soeroes, S.; Rajavelu, A.; Dhayalan, A.; Bock, I.; Rathert, P.; Brandt, O.; Reinhardt, R.; Fischle, W.; et al. Chromatin methylation activity of dnmt3a and dnmt3a/31 is guided by interaction of the add domain with the histone h3 tail. Nucleic Acids Res. 2010, 38, 4246-4253.

32. Dhayalan, A.; Rajavelu, A.; Rathert, P.; Tamas, R.; Jurkowska, R.Z.; Ragozin, S.; Jeltsch, A. The dnmt3a pwwp domain reads histone 3 lysine 36 trimethylation and guides DNA methylation. J. Biol. Chem. 2010, 285, 26114-26120.

33. Latos, P.A.; Pauler, F.M.; Koerner, M.V.; Senergin, H.B.; Hudson, Q.J.; Stocsits, R.R.; Allhoff, W.; Stricker, S.H.; Klement, R.M.; Warczok, K.E.; et al. Airn transcriptional overlap, but not its lncrna products, induces imprinted igf2r silencing. Science 2012, 338, 1469-1472.

34. Zhao, J.; Ohsumi, T.K.; Kung, J.T.; Ogawa, Y.; Grau, D.J.; Sarma, K.; Song, J.J.; Kingston, R.E.; Borowsky, M.; Lee, J.T. Genome-wide identification of polycomb-associated rnas by rip-seq. Mol. Cell 2010, 40, 939-953.

35. Sun, B.K.; Deaton, A.M.; Lee, J.T. A transient heterochromatic state in xist preempts $\mathrm{x}$ inactivation choice without rna stabilization. Mol. Cell 2006, 21, 617-628.

36. Storck, T.; Kruth, U.; Kolhekar, R.; Sprengel, R.; Seeburg, P.H. Rapid construction in yeast of complex targeting vectors for gene manipulation in the mouse. Nucleic Acids Res. 1996, 24, 4594-4596.

37. Nagy, A.; Rossant, J.; Nagy, R.; Abramow-Newerly, W.; Roder, J.C. Derivation of completely cell culture-derived mice from early-passage embryonic stem cells. Proc. Natl. Acad. Sci. USA 1993, 90, 8424-8428.

38. Evans, E.P.; Beechey, C.V. Personal Communication; MRC Mammalian Genetics Unit: Harwell, Oxfordshire, UK, 2010.

39. Lewandoski, M.; Meyers, E.N.; Martin, G.R. Analysis of fgf8 gene function in vertebrate development. Cold Spring Harb. Symp. Quant. Biol. 1997, 62, 159-168.

40. Lewandoski, M.; Martin, G.R. Cre-mediated chromosome loss in mice. Nat. Genet. 1997, 17, 223-225.

41. Truett, G.E.; Heeger, P.; Mynatt, R.L.; Truett, A.A.; Walker, J.A.; Warman, M.L. Preparation of pcr-quality mouse genomic DNA with hot sodium hydroxide and tris (hotshot). BioTechniques 2000, 29, 52, 54.

42. Shimomura, K.; Galvanovskis, J.; Goldsworthy, M.; Hugill, A.; Kaizak, S.; Lee, A.; Meadows, N.; Quwailid, M.M.; Rydstrom, J.; Teboul, L.; et al. Insulin secretion from beta-cells is affected by deletion of nicotinamide nucleotide transhydrogenase. Methods Enzymol. 2009, 457, 451-480.

(C) 2015 by the authors; licensee MDPI, Basel, Switzerland. This article is an open access article distributed under the terms and conditions of the Creative Commons Attribution license (http://creativecommons.org/licenses/by/4.0/). 